\title{
ASCA OBSERVATION OF THE CYGNUS LOOP SUPERNOVA
}

\section{REMNANT}

\author{
E. MIYATA AND H. TSUNEMI \\ Department of Earth and Space Science, \\ Graduate School of Science, Osaka University, Japan \\ CREST, Japan Science and Technology Corporation (JST)
}

\section{Introduction}

The Cygnus Loop is the prototype shell-like supernova remnant (SNR) and one of the brighest SNRs in X-ray wavelength. We have observed the entire Cygnus Loop with the X-ray satellite, ASCA. Its large apparent size, high surface brightness, and low absorption features have made the Cygnus Loop to be an ideal target for the study of the spatially-resolved spectroscopic structure in detail. Part of this work was summarized in Miyata (1996). Here, we present the first X-ray image of the Cygnus Loop obtained with ASCA.

\section{Observation}

ASCA observations were performed from PV-phase (1993/04) to AO-5 $(1997 / 06)$. The total number of observations is 30 and mean observing time is $\simeq 10 \mathrm{ks}$. The preliminary mosaic image obtained with the ASCA GIS is shown in figure 1 . We see some aritificial structures since the background component did not properly subtracted and vignetting effect was not corrected. Generally speaking, the limb-brightening structure can clearly be seen in this figures as previously shown in the Einstein image ( $\mathrm{Ku}$ et al. 1984 ) or in the Rosat image (Aschenbach 1994). Comparing with these softband images, we can find moderately strong emission from inner part of the Cygnus Loop. The most significant difference is the bright $\mathrm{X}$-ray compact source appeared at southern blow region of the Cygnus Loop. The flux of the source is $7.2 \times 10^{-12} \mathrm{erg} / \mathrm{s} / \mathrm{cm}^{2}$ and the ASCA spectra were well fitted with the absorbed power-law with photon index of $-2.1 \pm 0.1$ (Miyata et al. $1997 \mathrm{a})$. 


\section{Si Map}

By using the energy resolving power of the GIS, we investigated the extent: of heavy elements. Since Si lines are well resolved in the GIS spectra, we extracted the narrow line images of $\mathrm{Si}(1.6-2.1 \mathrm{keV})$ as well as the continuum image between $\mathrm{Mg}$ lines and $\mathrm{Si}$ lines (1.4-1.6 keV). The equivalent width map of Si can be constructed by dividing the Si image with the continuum image shown in figure 2. Equivalent width depends on $\mathrm{kTe}, \log \tau$, and abundance of heavy elements. Based on the detail studies of equivalent widths of $\mathrm{Si}$ at the center portion, Mivata et al. (1997b) suggested that the maximum equivalent width of $s i$ lines was $\simeq 0.9 \mathrm{keV}$ for the cosmic plasma. Therefore, figure 2 reirects the abundance distribution of Si. Based on figure 2, we can find that $\mathrm{Si}$ distributes from center portion toward the southern blow region. This suggests that ejecta is still confined inside the shell and has not yet mixed with the shocked interstellar medium.

At the southern blow region, the equivalent width is fairly high. Since the X-ray surface brightness of this region is quite low, the density of the interstellar medium might be low. This suggets that ejecta could be observable without any pollusion of the shocked interstellar medium.

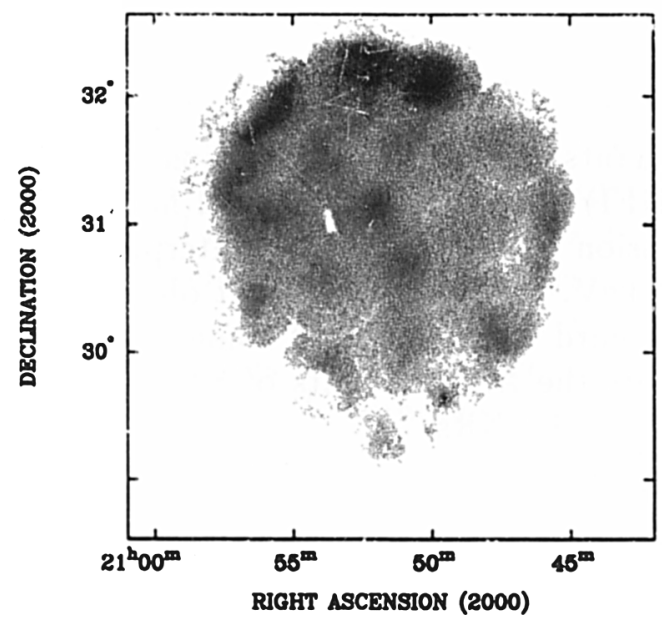

Figure 1. The X-ray image of the Cygnus Loop obtained with the ASCA/GIS

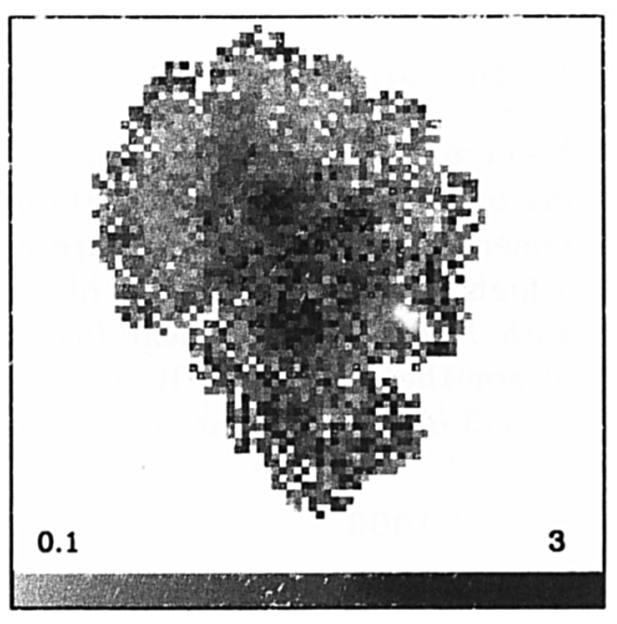

Figure 2. The equivalent width map of Si obtained with the ASCA/GIS in unit of $\mathrm{keV}$

\section{References}

Aschenbach, B. 1994, New Horizon of X-ray Astronomy, p.103

Ku, W.H.-M. et al. 1984, ApJ, 278, 615

Miyata, E 1996, Ph D. thesis, Usaka University

Miyata, E. et al. 1997a, PASJL submitted

Mivata. F, et al $1997 \mathrm{~b}$ PACI submittor 